\title{
Swarming in Bounded Domains
}

\author{
Dieter Armbruster \\ School of Mathematical and Statistical Sciences \\ Arizona State University \\ Sébastien Motsch \\ School of Mathematical and Statistical Sciences \\ Arizona State University \\ Andrea Thatcher \\ School of Mathematical and Statistical Sciences \\ Arizona State University
}

\begin{abstract}
The Vicsek model is a prototype for the emergence of collective motion. In free space, it is characterized by a swarm of particles all moving in the same direction. Since this dynamic does not include attraction among particles, the swarm, while aligning in velocity space, has no spatial coherence. Adding specular reflection at the boundaries generates global spatial coherence of the swarms while maintaining its velocity alignment. We investigate numerically how the geometry of the domain influences the Vicsek model using three type of geometry: a channel, a disk and a rectangle. Varying the parameters of the Vicsek model (e.g. noise levels and influence horizons), we discuss the mechanisms that generate spatial coherence and show how they create new dynamical solutions of the swarming motions in these geometries. Several observables are introduced to characterize the simulated patterns (e.g. mass profile, center of mass, connectivity of the swarm).
\end{abstract}

Keywords: Swarm models, Agent simulations, Dynamics of swarms PACS: 05.65.+b, 45.50.Jf, 87.23.Cc

Email addresses: armbruster@asu.edu (Dieter Armbruster), smotsch@asu.edu (Sébastien Motsch), andee.thatcher@gmail.com (Andrea Thatcher)

Preprint submitted to Elsevier

November 22, 2016

(C) 2016. This manuscript version is made available under the Elsevier user license http://www.elsevier.com/open-access/userlicense/1.0/ 


\section{Introduction}

Inspired by the observation of collective motion among a vast spectrum of biological organisms, researchers from several disciplines experimentally seek to understand the underlying properties governing coordinated movement. Many theoretical models have been introduced to capture such motion and aggregation.

Taking on this challenge, many disciplines for the past few decades have derived and analyzed self-propelled particle (SPP) models where individual particles only perceive nearest neighbors or all particles within an influence horizon rather than the entire group $[2,3,5,6,7,11,19,20,21]$. Among these SPP models, the Vicsek Model [21] has attracted a lot of attention. The Vicsek Model introduces a simple discrete-time algorithm where particles interact by aligning their velocity with their neighboring particles. As a result, collective behavior emerges and particles moves in a common direction measured by an observable called polarization. As the Vicsek model does not include attraction or repulsion, the dynamics do not create spatial coherence (particles tend to distribute uniformly in space). However, it is common experience that most swarms moving in real space show a spatial coherence in addition to the common direction of motion. Several extension have been proposed to include spatial organization through attraction interaction [15,4]. Another possibility, developed in this paper, is to include spatial coherence through the effect of boundary conditions.

Few studies have been conducted on the effects of boundaries on the Vicsek Model: Czirók and Vicsek [8] study swarming in both a circular and hexagonal domain and find that a rotation of particles develops for high densities and low noise levels. The direction of the rotation is random creating a single vortex regardless of the system size. Ouellette et al. [17] view the animal aggregate as a type of material and test how the three dimensional material breaks apart upon impact with a solid surface. They find that the initial particle mass fragments into smaller particle clusters obeying powerlaw distributions. Motsch and Navoret [18] have analyzed the tendency for the swarm to align in a torus with increasing lengths. For small noise levels, they found little influence of the torus size and a tendency to stronger alignment for large noise levels as the torus gets smaller.

At the same time, many swarms live in bounded domains: Typically all lab experiments like $[17,14]$ have finite sized domains. Similarly, even fish and bird swarms in nature are bounded above and below by one hard 
boundary and another soft boundary even though they often move in two unbounded dimensions. Other types of swarms like pedestrians and cattle are often guided (herded) in man-made channels. As those channels change directions, the swarms naturally interact with the boundaries.

In this paper, we show that the swarms generated by the Vicsek Model, if confined within a bounded domain with spectrally reflecting boundaries, have spatial coherence without additional attraction forces. For instance, we find new types of collective motion in addition to the single vortex discussed in [8] and analyze their behavior as a function of the noise level and the influence horizon.

Specifically, we focus on the time-continuous Vicsek Model derived in [10]. As a baseline we study the time evolution of an initially dense group of particles with random initial velocities and observe their swarming motion. We consider the dynamics in four different domains (see Fig. 1). First, we consider a square domain with periodic boundary conditions (Fig. 1A). It is the usual setting for most studies of this particular model. Then, we add vertical walls with reflective boundary conditions at the left and right side (Fig. 1B). We keep periodic boundary conditions in the vertical direction. The walls act as specularly reflecting flat surfaces for individual particles, i.e. the angle of incidence equals the angle of reflection. For the standard parameters of swarming in the Vicsek Model, the swarm has two long-time solutions that depend on initial conditions:

- A stationary flow in the direction of the channel that is homogeneous in space and denoted as the homogeneous flow solution. Since the flow is essentially parallel to the boundary, the vertical boundaries have little effect on the swarming behavior and the stationary velocity distribution follows the von Mises distribution derived in in [18] for periodic boundary conditions.

- A time dependent solution where the particle flow oscillates between a high density flow upwards at one boundary and a high density flow downwards at the other boundary called the shear flow solution. The shear flow solution is generated by a sticky boundary layer where the particle velocity aligns with the boundary. Particles moving towards the boundary align with the boundary layer, increase the density in the boundary layer and, thus, slow down the movement towards the boundary. In section 2.2 we discuss in detail how the flow towards the boundary reverses and leads to a periodic oscillation. We measure both 
the period of the oscillation and the width of the boundary layer length and show that the boundary layer length increases for increasing noise levels while the period decreases. On the other hand, the boundary layer length decreases while the period increases as the influence horizon increases.

As a third domain, we study swarms in a disk (see Fig. 1C). The typical long term solution becomes a spatially localized swarm that rotates close to the boundary of the disk. When we average the density of the particles over a long time, we find that the density profile on the disk is rotationally symmetric and is monotonically increasing towards the boundary of the disk. The density profile is similar to a steady state called the mill solution found as a solution partial differential equations (PDE) forming the macroscopic Vicsek model discussed in [18]. In fact, the mill solution is a steady state of the PDE written in polar coordinate in all of $\mathbb{R}^{2}$ and, thus, has an infinite total mass. Restricting the mass to a finite value inside the disk, the mill solution becomes indistinguishable from the averaged density of the rotating swarm. Making this identification, we numerically confirm that the mill solution is attracting, an issue that is still unresolved theoretically.

Finally, we study swarms in square domains (see Fig. 1D). The analytic mill solution is no longer a solution as the domain is not invariant by rotation. We study the dynamics for a wide range of swarm parameters and produce an averaged density profile similar to the circular domain. The associated path of the center of mass of the swarm has a $C^{4}$ symmetry and breaks the $D_{4}$ symmetry in the same way as the swarm in the disk breaks the $O(2)$ symmetry of the disk to an $S O(2)$ symmetry. The trajectory of the center of mass becomes predictable, associated with a very well defined periodic behavior of the swarm with high polarization. Changing swarm parameters, we find that the polarization of the swarm declines and, simultaneously, the trajectory of the center of mass of the particles becomes unpredictable, eventually leading to Brownian motion of the particles.

The outline of this paper is as follows: we introduce the discrete-time Vicsek Model along with several continuum versions in section 1.1. In 1.2 we discuss the simulation setup. In sections $2.1-2.3$ we present and analyze the simulations for various domains in $\mathbb{R}^{2}$. We conclude with a discussion in section 3 . 
A) Periodic

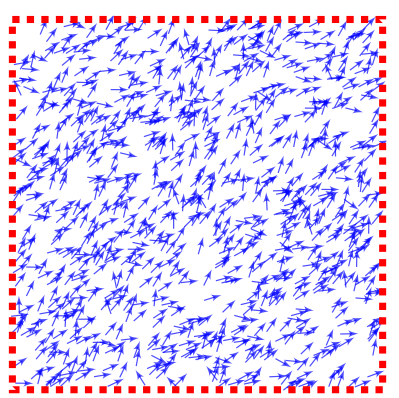

C) Disc

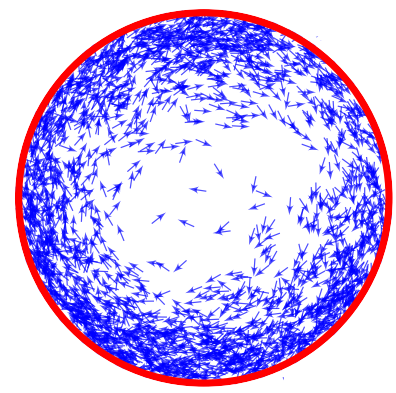

B) Channel

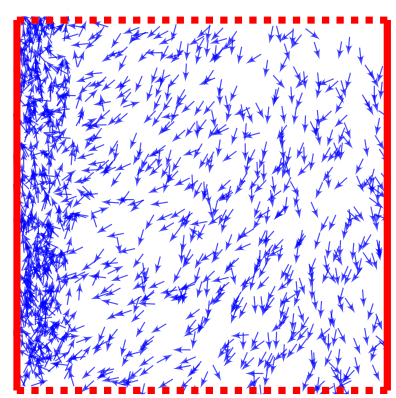

D) Square

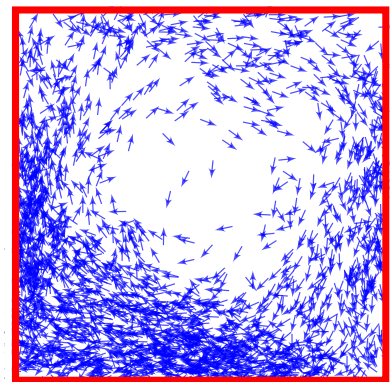

Figure 1: Four different settings for the numerical investigation of the Vicsek model. A) square domain with periodic boundary conditions (dashed lines), B) channel with periodic boundary conditions with reflecting boundary conditions in $x$ (solid lines), C,D) circle and square with only reflective boundary conditions. See also table 1 for the parameters used in the simulations. 


\subsection{Vicsek Model}

The time-discrete Vicsek Model proposed by [21] is considered the simplest self-propelled model exhibiting collective motion. Particles in this model are subject to a constant speed and interact by aligning their velocity direction to a local average velocity direction with an addition of noise. The position of each particle in a $d$-dimensional space is updated by adding the distance traveled during the time step in the direction of its velocity angle.

Specifically, the position of the $k$ th particle at a discrete time $n \Delta t$, denoted $x_{k}^{n}$, for $k \in\{1 \ldots N\}$ where $N$ is the total number of particles, is updated as

$$
x_{k}^{n+1}=x_{k}^{n}+w_{k}^{n} \Delta t .
$$

Its velocity $w_{k}^{n}$ evolves according to the average velocity at each time step $\bar{w}_{k}^{n}$ of the neighboring particles along with an addition of noise $\eta_{k}$. The average velocity is defined as:

$$
\bar{w}_{k}^{n}=\frac{J_{k}^{n}}{\left|J_{k}^{n}\right|} \quad, J_{k}^{n}=\sum_{j,\left|x_{j}^{n}-x_{k}^{n}\right|<R} w_{j}^{n} .
$$

Notice that the velocity average $\bar{w}_{k}$ is taken over the neighboring particles to particle $k$ within a radius $R>0$ ball centered at the position $x_{k}^{n}$. The velocity is updated according to:

$$
w_{k}^{n+1}=\frac{w_{k}^{*}}{\left|w_{k}^{*}\right|} ; \quad w_{k}^{*}=\bar{w}_{k}^{n}+\eta_{k} .
$$

The noise values $\eta_{k} \in \mathbb{R}^{d}$ are typically assumed to be independent random variables with uniform distribution in $[-a, a]^{d}, a>0$. The Vicsek Model assumes a constant speed hence the normalization in (3).

A time continuous version of the dynamics has been developed in [10]; it consists of the following coupled differential equations:

$$
\begin{aligned}
\frac{d x_{k}}{d t} & =w_{k} \\
d w_{k} & =P_{w_{k}^{\perp}}\left(\nu \bar{w}_{k} d t+\sqrt{2 d} d B_{t}\right)
\end{aligned}
$$

where $P_{w_{k}^{\perp}}=\left(\mathrm{Id}-w_{k} \otimes w_{k}\right)$ is the orthogonal projector onto the plane normal to $w_{k}$, ensuring that the velocity vector is an element of the unit sphere (i.e. $\left.w_{k} \in \mathbb{S}^{2}\right), d B_{t}$ is Brownian motion with intensity $\sqrt{2 d}$ and $\bar{w}_{k}$ 
is the continuous velocity average in a ball of size $R$ equivalent to (2). The interaction frequency $\nu$ and the diffusion coefficient $d$ replace the time step $\Delta t$ and noise level $\eta_{k}$. Hence $\nu^{-1}$ is the typical decay time for perturbations of particles velocity to align with their neighbors.

When considering the hydrodynamic limit of the dynamics by rescaling time and space as $t^{\prime}=\epsilon t$ and $x^{\prime}=\epsilon x$, one can show that the dynamics (4) lead to a set of partial differential equation for the evolution of two macroscopic quantities, the density of the particles $\rho$ and the direction of the particle flow $\Omega$ :

$$
\begin{aligned}
& \partial_{t} \rho+c_{1} \nabla_{x} \cdot(\rho \Omega)=0 \\
& \rho\left(\partial_{t} \Omega+c_{2}(\Omega \cdot \nabla) \Omega\right)+d P_{\Omega^{\perp}}\left(\nabla_{x} \rho\right)=0
\end{aligned}
$$

where $c_{1}$ and $c_{2}$ are constant coefficients depending on $d$ with $c_{1} \neq c_{2}$, the vector $\Omega$ is a direction and satisfies $|\Omega|=1$. We refer to this model as the macroscopic Vicsek Model [10].

\subsection{Simulation Setup}

We use Euler's method to compute the numerical approximation to the continuum Vicsek model (4). For Euler's method to remain stable, we require $\nu \Delta t<1$. When reaching the reflecting boundary, a particle $k$ will experience specular reflection and its velocity is updated according to

$$
w_{k} \quad w_{k}^{*}=w_{k}-2\left(w_{k} \cdot \vec{n}\right) \vec{n},
$$

where $\vec{n}$ is the normal vector to the boundary.

A particle $k$ interacting with the wall at time step $n \Delta t$ will not update its velocity according to Eq. (4) until the next time step $(n+1) \Delta t$. That is, we have a time step $\left(n+\zeta_{k}\right) \Delta t$ with $0<\zeta_{k}<1$ where the velocity $w_{k}^{n+\zeta_{j}}$ is reflected by Eq. (6) and the particle $k$ continues on this new trajectory until time step $n+1$. At this new time step $n+1$, the velocity updates according

to Eq. (4) with the reflected velocity $w_{k}^{n+\zeta_{j}}$ instead of the original velocity $w_{k}^{n}$.

As a result, different time steps satisfying the CFL condition create different transient results. We keep our time step $\Delta t=0.01$ to avoid conflicting results that depend on the time step.

The parameter choices for each simulation (unless stated otherwise) are: the time step $\Delta t=0.01 ; \nu=1$; the number of particles is $N=10,000$; and the radius of interaction is $R=0.5$ space unit. The domain size $L$ is typically 10 space unit, thus the disc in figure 1c) is of radius 5. Simulations 
are ran for various lengths of time. Most settled to the described behavior after an order of 100 time units. We ran most tests ten times longer to verify the behavior. A summary of the parameters used and their values are given in the table 1.

Simulations were run using gfortran on 96gb RAM and 2 Intel(R) Xeon(R) CPU E5-2620 0 @ 2.00GHz processors and took an average of 600 seconds to run 100 time units.

\begin{tabular}{l|l|r}
\hline Number of particles & $N$ & 10,000 \\
alignment rate & $\nu$ & 1 \\
noise level & $d$ & $0-5$ \\
radius observation & $R$ & 0.5 \\
domain size & $L$ & 10 \\
time step & $\Delta t$ & .01 \\
time simulation & $T$ & $100-1,000$ \\
\hline
\end{tabular}

Table 1: Parameters used for the simulations along their value or range.

\section{Swarms in bounded domains}

We show, via simulation studies of the continuum Vicsek model, that if swarms are confined within a bounded domain with spectrally reflecting boundaries, spatial coherence without additional attraction forces is produced. We also find new types of collective motion and analyze their behavior as a function of the noise level and the influence horizon for different spatial domains.

\subsection{Periodic Boundary Conditions}

In a square domain with periodic boundary conditions, the continuous Vicsek model leads to a flocking type behavior where the agents moves preferably in a common direction as illustrated in the figure 1a). The flocking aspect of the swarm is governed by the level of noise $d$. To further investigate the alignment of the flock, a common observable used is the average velocity

$$
\phi=\frac{1}{N}\left|\sum_{k=1}^{N} w_{k}^{n}\right| .
$$

$\phi \approx 1$ implies particles behave like a swarm and all move in the same direction and $\phi \ll 1$ implies the particles move randomly with no ordered motion. 
In a spatially homogeneous setting, one can prove $[10,13,9]$ that the distribution of velocities converges to a so-called von Mises distribution

$$
M_{\Omega}(\omega)=Z \exp \left[\frac{\omega \cdot \Omega}{d}\right]
$$

where $\Omega$ is the direction of the mean velocity of the particles and $Z$ is a normalizing constant. This result implies that for a homogeneous distribution of particles the average velocity has the analytic expression

$$
\phi_{\text {homog }}=\left|\int_{\omega \in \mathbb{S}^{1}} \omega M_{\Omega}(\omega) d \omega\right| .
$$

In Figure 2, we compare both the estimations of $\phi(7)$ and the analytic expression (9) for various noise level $d$. We observe that the velocity average $\phi$ matches nicely with the explicit value $\phi_{\text {homog }}$ for low noise level $d$ (i.e. $d \lesssim 1.5)$. Then the curve $\phi$ has a sharp decay around $d=1.5-2$ whereas $\phi_{\text {homog }}$ decays smoothly. This difference in the transition between flocking behavior (i.e. $\phi \approx 1$ ) and non coherent dynamics (i.e. $\phi \approx 0$ ) has been the source of a large debate in the physics community $[21,16,1,22]$. It is still undecided whether the Vicsek model has a continuous or second order phase transition.

Since there exists no common velocity direction for large noise levels, it is suitable to introduce a local estimation of the alignment. To do so, we introduce the localized version of the estimator $\phi$ :

$$
\phi_{\text {local }}=\left\langle\frac{1}{N_{k}}\left|\sum_{\left|x_{j}^{n}-x_{k}^{n}\right|<R} w_{j}^{n}\right|\right\rangle, \quad N_{k}=\#\left\{\left|x_{j}^{n}-x_{k}^{n}\right|<R\right\},
$$

where $\langle$,$\rangle denotes the averaging over all particles k, R$ is the radius of observation of the Vicsek model and $N_{k}$ is the number of neighbors around $k$. We illustrate the estimator $\phi_{\text {local }}$ in Fig. 2. We observe that the decay of $\phi_{\text {local }}$ matches with the curves of $\phi_{\text {homog }}$ for all noise levels. As a consequence, we can argue that the global alignment $\phi$ does not only reflect the internal dynamics the Vicsek model but also encompass global spatial configuration.

\subsection{Channel Domain}

In our second setting, we introduce specular reflection at the left and right side of the domain, i.e. we add two walls at $x=0$ and $x=L$. The domain 

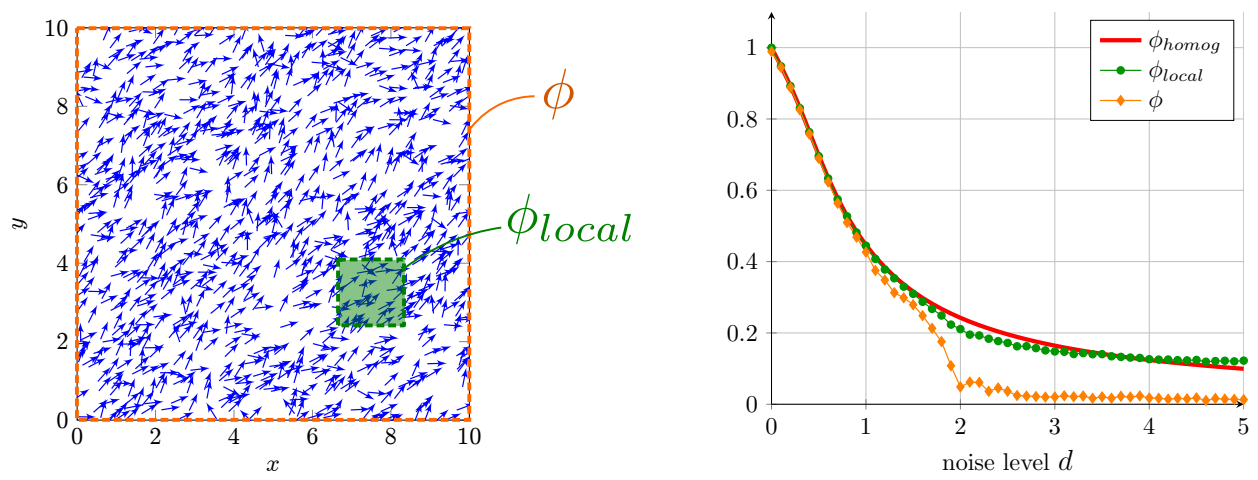

Figure 2: Left: Simulations of the Vicsek model in a square domain with periodic boundary conditions after $T=100$ unit times with the noise level $d=.2$. Although initially the velocity particles are uniformly distributed, the particles converge to a common direction. Right: Estimation of the global and local alignment coefficient (resp. $\phi$ and $\phi_{\text {local }}$ ) along with the analytic homogeneous formula (i.e. $\phi_{\text {homog }}$ ) for various noise levels $d$. For small noise levels (i.e. $d \in[0,1.5]$ ), all estimations agree. In contrast, for $d>1.5$, the global estimation $\phi$ drops indicating that there is no more flocking behavior for those noise levels.

is therefore a one-dimensional channel with $x$ as the transverse direction and $y$ as the parallel direction (see Fig. 1B). This modification allows to observe new type of behavior for the swarm, namely an oscillatory solution moving back and forth between the two walls.

To observe such oscillatory solution, we set up the initial condition as a Riemann problem assuming a uniformly density distribution in space, denoted $\rho(x, y)$, and an initial velocity direction for the particles that differ for the right and left half of the channel. In the left half of the channel, i.e for positions $0 \leq x<L / 2$, the velocity of the particles are distributed in the direction $\Omega_{L}$, whereas in the right side of the channel, i.e. for positions $L / 2<x \leq L$, the mean direction of the velocity is given by $\Omega_{R}$. The vectors $\Omega_{L}$ and $\Omega_{R}$ are chosen with an anglular direction of $\theta_{0}$ and $-\theta_{0}$ resp., i.e. $\Omega_{L}=\left(\cos \theta_{0}, \sin \theta_{0}\right)$ in the basis $\left\{\mathbf{e}_{x}, \mathbf{e}_{y}\right\}$. This configuration is depicted on Figure 3-upper-left with $\theta_{0}$. Rather than plotting all the particles, we represent the average density of particles $\rho$ (color coded) and the local average velocity $(u, v)$. More precisely, on a grid cell $\mathcal{C}_{i, j}$ of size $\Delta x \Delta y$, density and average velocities are given by:

$\rho_{i, j} \Delta x \Delta y=\#\left\{x_{k} \in \mathcal{C}_{i, j}\right\} \quad, \quad u_{i, j}=\left.\left\langle\cos w_{k}\right\rangle\right|_{x_{k} \in \mathcal{C}_{i, j}} \quad, \quad v_{i, j}=\left.\left\langle\sin w_{k}\right\rangle\right|_{x_{k} \in \mathcal{C}_{i, j}}$

where $\#\{$.$\} denotes the number of elements in the set, \left.\langle\rangle\right|_{.x_{k} \in \mathcal{C}_{i, j}}$ is the aver- 
aging over the elements in $\mathcal{C}_{i, j}$. We refer to [12] for more information about Particle-In-Cells methods.

Depending on the noise level $d$, there are two type of long term behaviors of the swarm. First, the swarm might converge to a homogeneous solution where the particles remain evenly dispersed in space and have all a common upward or downward motion. The homogeneous solution is the swarming solution discussed in the previous section. In the second type of solution, the particle flow oscillates between a high density flow upwards at the left boundary and a high density flow downwards at the right boundary. This time dependent state is named the shear flow solution and is illustrated in Figure 3 at different times.

Remark 1. The oscillatory solution can also emerge from an initial condition with a random (uniform) distribution of particles in both space and velocity. However, the homogeneous solution is more likely to occur from this initial condition. Starting from the configuration described above (i.e. Riemann problem) promotes the emergence of the shear flow solution.

Since shear flow solutions emerge only for certain initial conditions, we vary the two parameters $\theta_{0}$ (velocity direction) and $d$ (level noise) and estimate when the dynamics converge to either a homogeneous solution or to a shear flow solution. As a result of this exploration, we obtain a bifurcation diagram (see Fig. 4).

To understand the details of the shear flow, we study the interaction of an arriving swarm at the right boundary in several steps:

1. Assume that particles at the right boundary are moving downward, i.e. $u(x=10, y)=0, v(x=10, y)=-1$ (see Fig. 3,top-right). As a result the transverse velocity is zero and the particles are not leaving the boundary. Consider a swarm moving upward that is approaching the right boundary, i.e. all particles have a positive velocity in the $x$ direction moving. The 'front' of the swarm, denoted $x_{*}$, is defined as the transition between upward and downward velocity, i.e. $v\left(x_{*}\right)=0$ (see also Fig. 5-left). Initially, the swarm is moving right so is the front position $x_{*}$.

2. Due to the alignment interaction, the norm of the velocity $(u, v)$ should remains close 1 , therefore at the front position $x_{*}$, the transverse velocity $u$ is maximum (since $v=0$ ). Thus, the dynamics generate a 

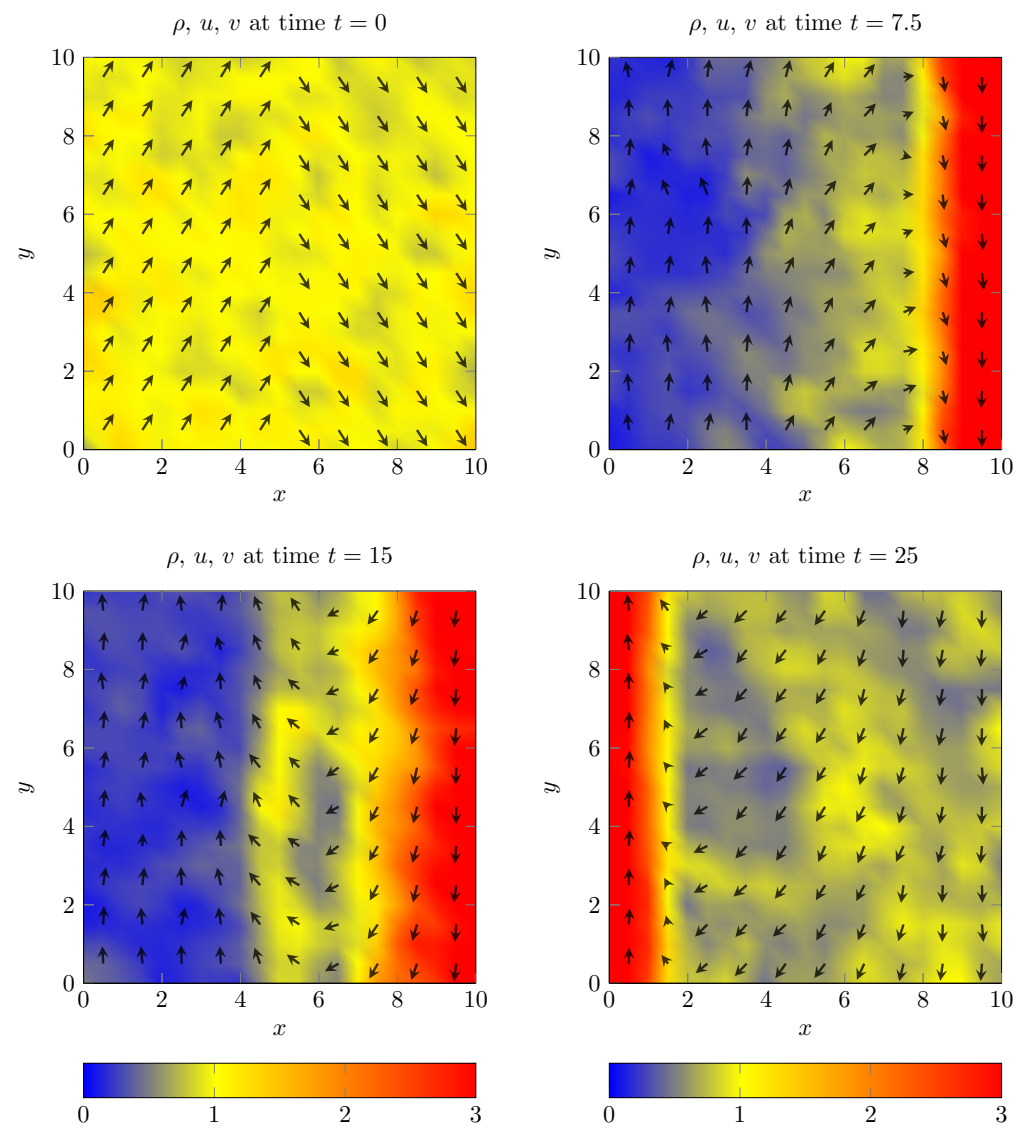

Figure 3: Time progression of the particle density and velocity plots for the shear flow solution. The average density in each cell is represented by a color code where yellow implies low density and black implies high density. The average velocity is represented by an arrow in the center of the cell. The noise level $d=0.2$ and the initial angle $\theta_{0}=1$. Other parameters are given in table 1. 
compression of particles in front of $x_{*}$ and a dilution of particles behind $x_{*}$.

3. As a result the density at the right boundary is increasing and so is the internal pressure. When the front $x_{*}$ is close enough to the wall, there is no more influx of particles to counterbalance the pressure resulting in a wave of particles leaving the wall to the left. A density depression trailing the wave ensures that eventually a significant number of particles will be stuck at the right wall and will not participate in the swarm movement to the left.

Since shear flow solution emerge only for certain initial condition, we vary the two parameters $\theta_{0}$ (velocity direction) and $d$ (level noise) and estimate when the dynamics converge to either a homogeneous solution or to a shear flow solution. In Figure 4, we estimate how frequent shear solution occur depending on $\theta_{0}$ and $d$. Shear solution do not occur if the noise level is too large (e.g. $d \geq 1.5$ ) or if the angle $\theta_{0}$ is too small (e.g. $\theta_{0} \leq .1$ ). Surprisingly, shear flow is also less frequent with small noise (with $0 \leq \theta_{0} \leq 0.4$ ). The explanation is that the particle at the left boundary (i.e. $x=0$ ) all left the boundary if the noise is too small. Therefore, there is no more particles heading upward and the left wall loses the information of the initial condition.

To investigate the shear flow solution further, we characterize the period of the oscillations and the size of the boundary layer that forms (periodically) at the left and right side of the channel. For instance, on figure 3 at $t=$ $7.5,15,25$, the boundary layer seems to be of order 2 space unit. To estimate more precisely such quantity, we are interested at the transition point on the $x$-axis where the flow of particles is moving upward and downward. Taking a cross-section in $x$ in Figure 5-left, the transition point $x_{*}$ satisfies $v\left(x_{*}\right)=0$, i.e. particles are neither moving up or down. The evolution of this point $x_{*}$ in time is given on Fig. 5-right, this curve provides an estimation for both the period of the solution and the size of the boundary layer: the period of the solution is given by the period $\tau$ of the curves, the size of the boundary layer $\ell$ is the minimum distance between the transition point $x_{*}$ and each wall (i.e. $\left|x_{*}\right|$ and $\left|x_{*}-10\right|$ ). The estimations of $\tau$ and $\ell$ are performed through a Fourier analysis, we fit the evolution of $x_{*}(t)$ with a wave function (red curve in Fig.5-right) and deduce its periodicity and minimum distance with the walls (Fig. 5-right).

We iterate this procedure to estimate the periodicity $\tau$ and boundary layer size $\ell$ depending on the noise level $d$. In fFgure 6 and for each value 

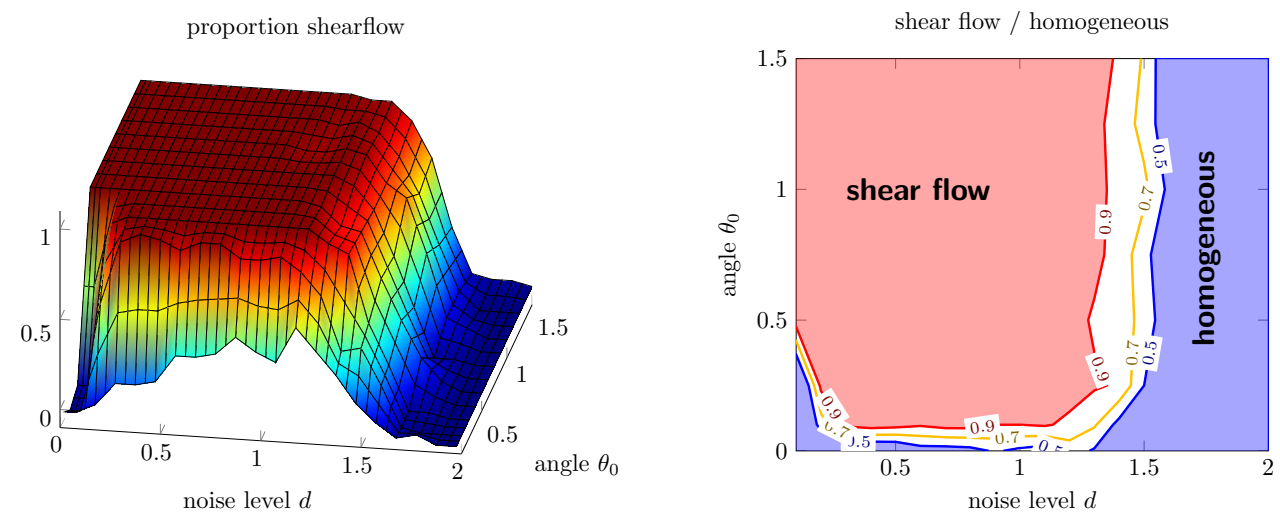

Figure 4: Bifurcation diagram of the parameters $\theta_{0}$ and $d$ for the initial velocity distributions illustrated in figure 3-top-left. For each value of $d$ and $\theta_{0}$, we run 40 simulations and estimate the frequency of shear flow solution. The simulations run for 100 time units. Left: the proportion of shear flow as a function of $d$ and $\theta_{0}$ is represented. Right: contour plot for the same distribution. The red region indicates where the proportion of shear flow is higher than $90 \%$ and in blue where the proportion is lower than $50 \%$.

of $d, 30$ realizations are performed after which the average values of $\tau$ and $\ell$ are computed. We observe in Figure 6-left that the period $\tau$ decreases as the noise $d$ increases. This observation appears counter-intuitive since at higher noise level, the local average velocity is lower (see Fig. 2) and hence one would expect the period to increase. However, one could also observe that the boundary layer $\ell$ is getting smaller for lower noise level $d$ (see Fig. 6-right) and, therefore, the swarm spends more time nearby the walls. Moreover, the wave traveling back and forth has to cover a shorter interval for larger noise and, hence, the period decreases as the noise increases.

We perform a similar procedure modifying the radius of the horizon $R$ of the particles. In contrast to varying $d$ (Fig. 6a), the period increases with $R$ (Fig. 7a). Essentially, as $R$ increases the velocity average is taken over more and more particles, generating inertia to change and thus lengthening the period of an oscillation. Thus, the scaling behavior of the period with respect to noise is anti-correlated with the scaling behavior with respect to $R$ : reducing $R$ is equivalent to increasing noise and vice versa. Similarly, we observe that increasing the horizon $R$ has the opposite effect for the size of the boundary layer (Fig. 7b): $\ell$ is decreasing as $R$ increases.

This anti-correlation suggests that if $R$ is small enough and $d$ large 

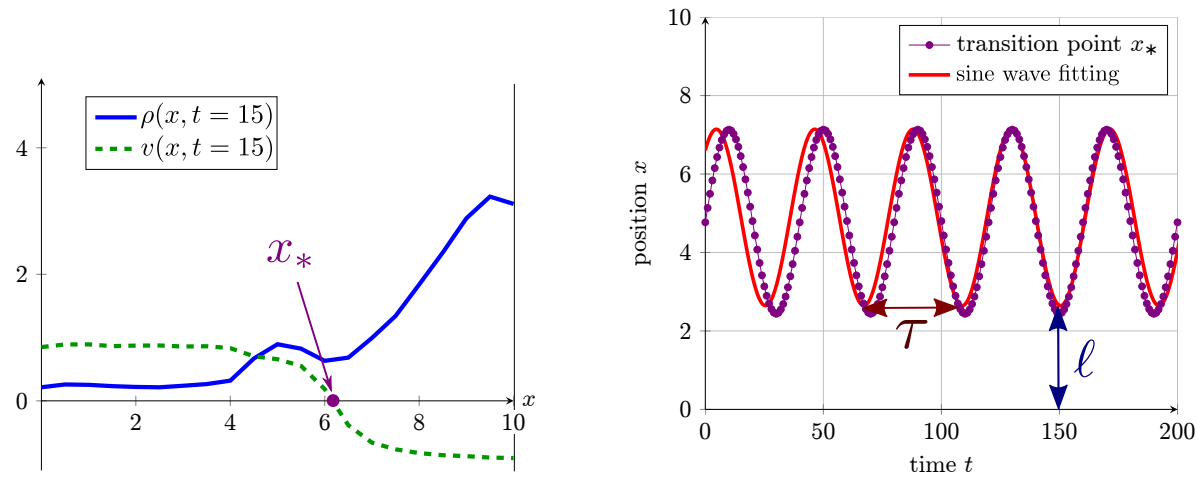

Figure 5: Left: Cross section in $x$ of the density $\rho$ and velocity $v$ in the $y$-direction. The transition point $x_{*} x \approx 6.2$ indicates where the particles switch from upward to downward motion. Right: Evolution of the transition point $x_{*}(t)$ over time (dot-green) and a fitting with a wave function (red) used to determine the size of the boundary layer and the periodicity of the solution.

enough, the instability will remove enough particles from the wall, such that they do not have enough influence any more to maintain the initially prescribed flow at the boundary, leading eventually to the homogeneous flow solution. Indeed, for $R=0.2, d>1.5$ the ratio of homogeneous to shear flow solutions for the initial direction angle $\theta_{0}=1$ increases (see Fig. 4).

\subsection{Circular Domain}

We consider swarming behavior in a disk of radius $R_{D}=5$ unit space. Initially, all particles are uniformly distributed within the disk and their initial velocities are also uniformly uniformly distributed. The typical long term behavior of the Vicsek model in this setting is a mill formation with a higher concentration of particle near the boundary of the disk (see Figure 1c).

This mill solution is actually an explicit solution of the macroscopic Vicsek Model. Indeed, in polar coordinates $(r, \theta)$, we can find an explicit solution given by:

$$
\rho(r, \theta)=C r^{\alpha} \quad, \quad \Omega(r, \theta)=(-\sin \theta, \cos \theta) .
$$

Here $r$ is the radius from the center of the domain, $C$ is a normalization constant and the power $\alpha$ depends explicitly on the coefficients $c_{1}, c_{2}$ and $d$ of the macroscopic Vicsek model. We refer to [18] for the explicit value of $\alpha$. 

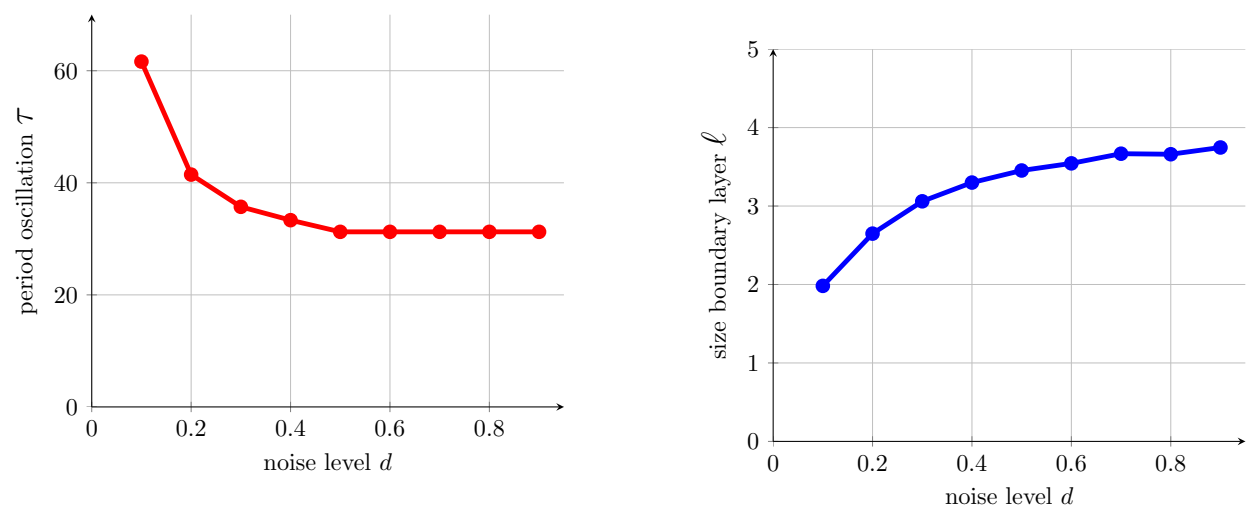

Figure 6: Periodic time and boundary layer thickness for various noise levels $d$. The periods (left) and boundary layers (right) are estimated from the sine wave fitting function (see Fig. 5). The simulations are run for 500 time units and an average over 30 realizations are performed for each value of $d$ (horizon $R$ is fixed and equal to .5).
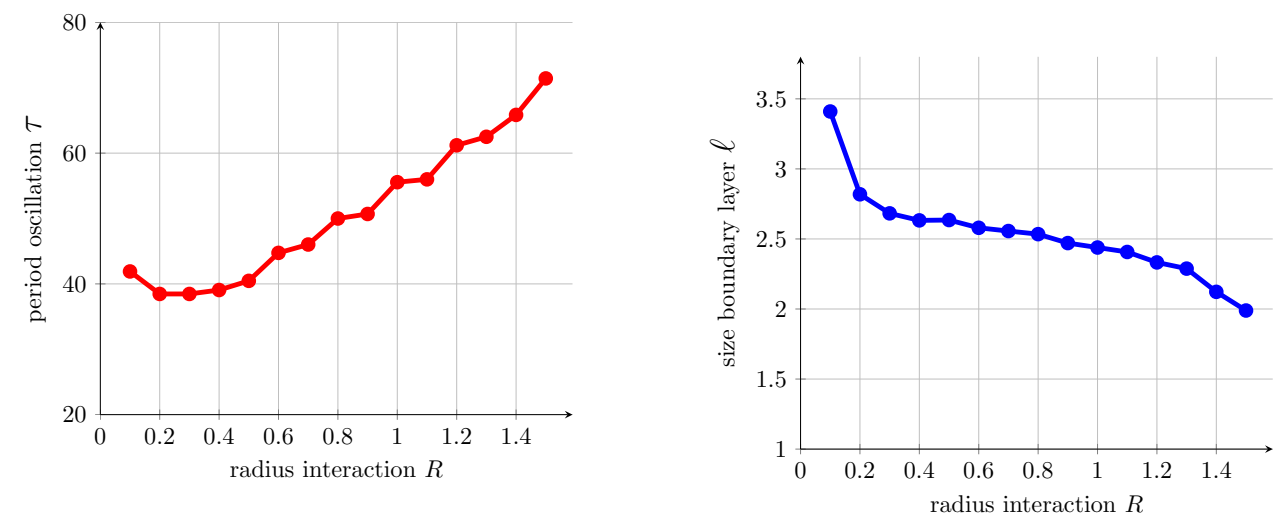

Figure 7: Periodic time and boundary layer thickness for various vision horizons $R$. The periods (left) and boundary layers (right) are estimated from the sine wave fitting function (see Fig. 5). The simulations are run for 500 time units and an average over 30 realizations are performed for each value of $R$ (the noise level $d$ is fixed and equal .2). 

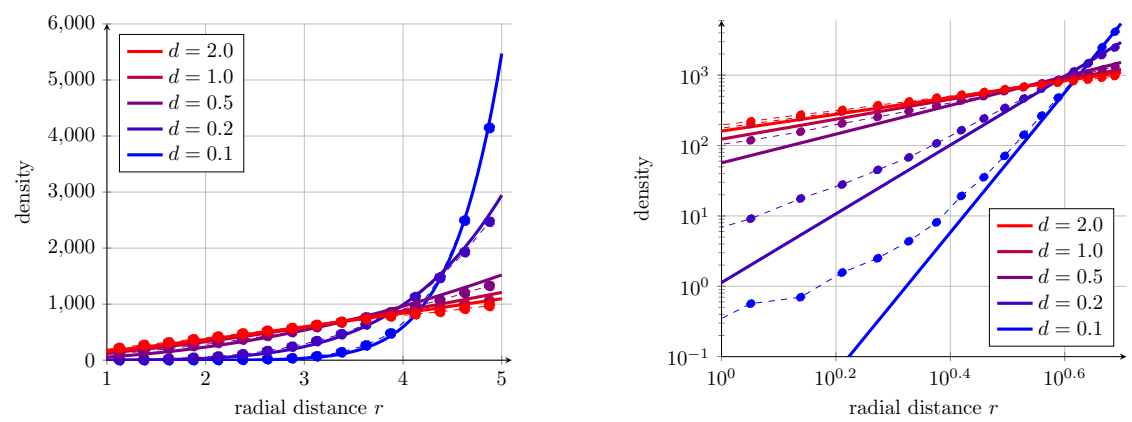

Figure 8: Left) Radial distribution of particles $\bar{\rho}(r)$ for the microscopic model (dots) and macroscopic model (line) with four different noise values $d$. The simulations run for 500 time units with the plotted values being averaged over 30 simulations. Right) Similar plot in log-log scale.

The constant $C$ can be explicitly determined as the total mass is conserved:

$$
\int_{\theta=0}^{2 \pi} \int_{\rho=0}^{R_{D}} \rho(r) r d r d \theta=1 \quad \Rightarrow \quad C=\frac{(\alpha+2) R_{D}^{-(\alpha+2)}}{2 \pi} .
$$

Notice that in the steady state considered in [18], there are no boundary conditions. Solutions of this form (12) rotate about the center of the domain with unit speed while the density profile grows without bound. As the mill solution has no flux through a concentric circle, it may be adapted to a finite circular disk with no-flux boundary conditions, i.e $n \cdot \Omega=0$ at the boundary where $n$ is the normal vector to the circle.

To compare the long-term particle behavior of the microscopic model to the mill solution of the macroscopic model (12), we compute the radial distribution of particles $\bar{\rho}(r)$ by estimating the density on concentric circle with radius $r$. In Fig. 8, we compare the radial distributions for both the microscopic and macroscopic Vicsek model (resp. dots and line) for several values of the noise $d$. We observe that the two curves match nicely with each other. For low noise, the density is compacted closely to the wall. Actually, without noise (i.e. $d=0$ ), the distribution of particles would be a Dirac mass at the boundary. In contrast, when the noise $d$ is large, the distribution becomes uniform in space.

Figure 8 indicates that the mill solution is attracting. An analytic proof of that fact is an open problem. The close match between the macroscopic 


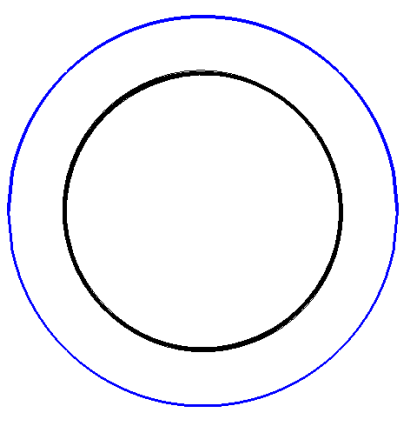

(a) Trajectory

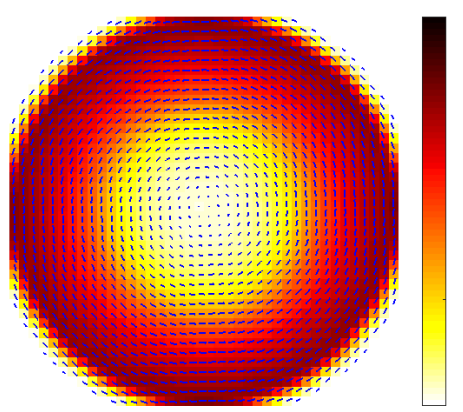

(b) Density

Figure 9: A rotating swarm in a disk. a) trajectory of the center of mass. b) time average of the density. Circle radius $R_{D}=5, d=0.1$, and all other parameters set to default. The simulations run for 1000 time units.

mill solution and the microscopic particle simulation is unexpected: While the mill solution (12) has a density distribution that rotationally invariant, the actual swarm moves as a localized blob around the circle as can be seen in Fig. 9a). The corresponding trajectory of its center of mass is a concentric circle, Fig. 9b). Averaging the motion of the blob over time, we obtain the density profile of the mill solution, Fig. 9c). This equivalence suggests that for stationary flows the time average of the particle simulation become the equilibrium solutions in the long time scaling that is the base of the macroscopic model. Note that the explicit solution Eq. (12) is valid for a disk of any size. Hence we expect to have a similar density profile and the equivalence between time average of the particle simulation and the equilibrium density for any size disk.

\subsection{Square Domain}

We study the long term behavior of the Vicsek Model in a square box of size $L=10$ unit space. All particles are initially uniformly distributed within the box with initial velocities uniformly distributed on the unit circle. Similar to our analyses of swarms in the disk, we measure the mass profile as

$$
\bar{\rho}(l)=\int_{\max \{|x|,|y|\}=\ell} \rho(x, y) d x d y,
$$

where $\rho(x, y)$ is the density of particles (11). Thus, $\bar{\rho}(l)$ computes the total number of particles on a square of length $l$ centered at the center of the 
domain. The density profiles (Fig. 10) show that particles are more highly concentrated near the boundary for smaller noise level $d$ and become more evenly dispersed as $d$ increases. Following the analysis for the disk, we show that the trajectory of the center of mass trajectory for these selected $d$ values (Fig. 11) is periodic with very little noise dispersion. The corresponding particle motion is a localized swarm that rotates about the center of the domain.

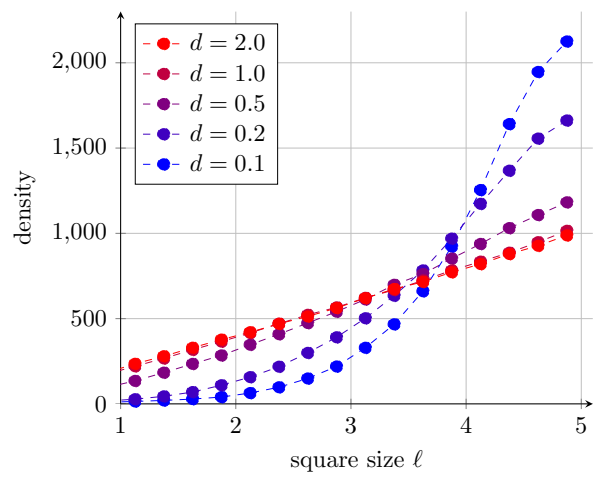

Figure 10: Total mass profile in the square for several noise values $d$. The curves from left to right correspond to a decreasing $d$. We plot Eq. (14) for rotating swarms in a square box. Number of particles $N=10000$, box size $L=10$, and $d=.1-2$. The other parameters are given in table 1 . The simulations run for 500 time units with the plotted values being averaged over 30 simulations.

As usual, when $d$ is large enough, the swarm loses coherence and the particles perform a random walk in the square, leading to a uniform density distribution. However, when the noise $d$ is small enough, but not equal to zero, the swarm also loses predictability and its center of mass follows a seemingly chaotic trajectory (Fig. 12b).

The reason a deterministic swarm shows a irregular trajectory can best be understood by a thought experiment: Assume a spatially extended swarm moves towards a horizontal boundary with uniform velocity normal to the boundary. When the leading edge of the swarm hits the boundary, most of the swam is still moving towards the boundary hence the average velocity of the swarm is still towards the boundary and therefore the leading particles get stuck at the boundary. The time when the swarm rebounds and leaves the boundary is determined by the ratio of the number of particles that 


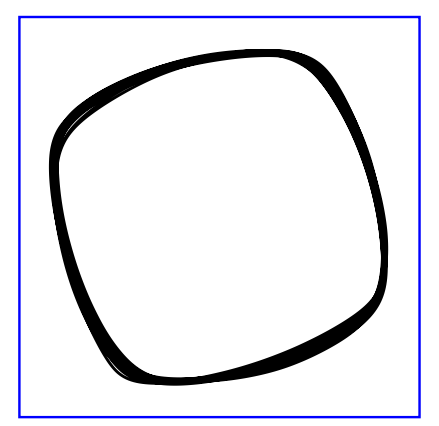

Figure 11: Trajectory for the center of mass trajectory of a swarm in a square. Parameters are set to figure 10 for $d=0.05$. The simulations run for 1000 time unit. The figure shows the last fifty time units.

hit the boundary within one time interval of the discrete update and the number of particles that do not hit the boundary in that time interval. If that ratio is above 1 , then the swarm leaves the boundary. When the ratio is approximately one, then the average velocity that determines the next direction of movement is almost zero and hence very susceptible to slight perturbations. Those might be a very low noise level or a particle that is at the periphery of the swarm and hits a sidewall of the box instead of a horizontal wall. Any of these small perturbations will be greatly amplified, since the velocity is normalized and hence the small velocity vector is divided by a normalization constant that is almost zero. As a consequence the motion of the swarm after its collision is hard to predict.

In figure 12a), we include the average velocity of the particles $\phi_{N}$ as a function of time. When the average velocity is small $\phi_{N}<0.35$, we see the chaotic situation where the particle Center of Mass follows no clear path (b). However, when $\phi_{N}>0.5$ in (c), we see a rotational path that is not as clearly defined as figure 11 .

When the particle mass approaches the boundary, the particle velocities average out to zero, giving the Gaussian noise the ability to overpower the contribution from the average velocity. When the velocity is normalized, the swarm has a random direction and fails to align with the boundary.

\section{Discussion}

We numerically analyzed the Vicsek Model in bounded domains and showed that the major effect of the boundaries is their stickiness which leads 


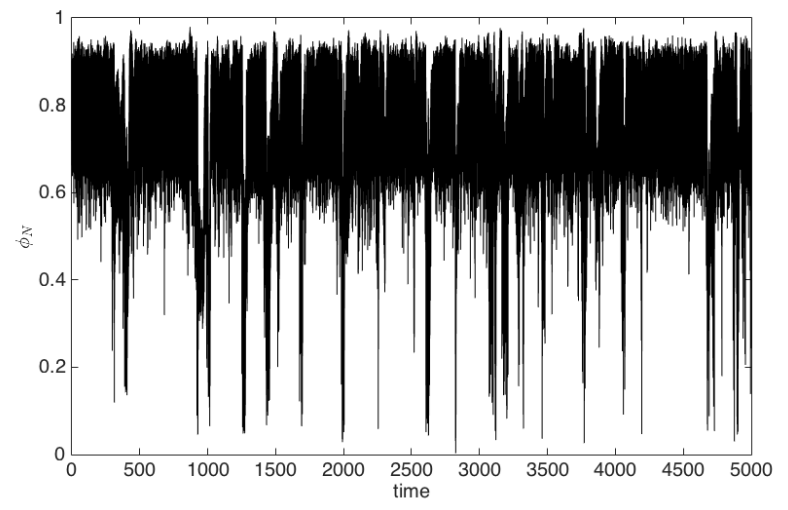

(a) $\phi_{N}$

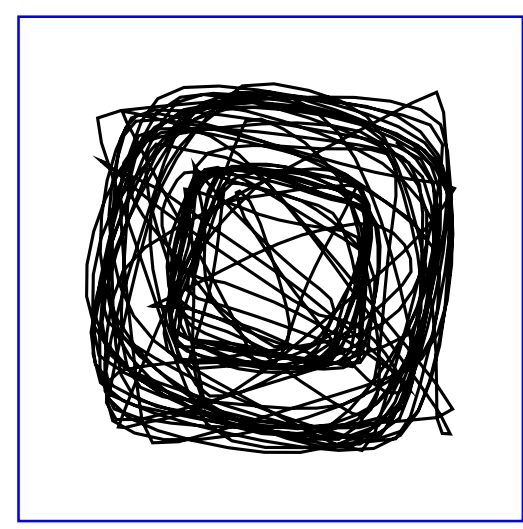

(b) Center Of Mass: $t=900$ to 1050. $\phi_{N}<0.35$

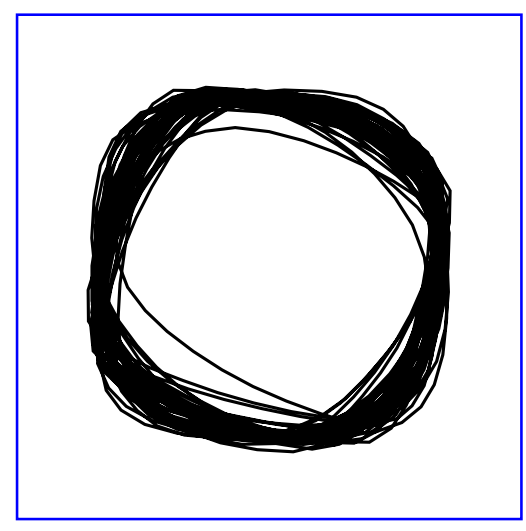

(c) Center Of Mass: $t=500$ to 650. $\phi_{N}>0.5$

Figure 12: Trajectory for the center of mass trajectory of a swarm in a square box. We initialize with the rotational state seen in figure 11 with the same parameters and decrease the noise level $d=0.035$. The simulation runs for 5000 time units. 
to spatial coherence of swarms. The lack of spatial coherence in the Vicsek Model was previously addressed by adding attractive forces. Our simulations suggest, that at least in finite domains of reasonable size, the boundaries may act as the attractors and hence no further forces are needed. In that sense, boundaries generate coherence of the swarm way beyond the obvious confinement due to the finite domains. In fact, the resulting swarms have a support, that is very localized, generating localized high densities in fractions of the actual domain size. In addition, the spatial coherence interacts with the movement of the swarm and generates space and time dependent dynamics that are not present in unbounded domains.

Specifically, we study three domains, 1-d channels, squares and disks. We assume specular reflection for the individual particle and show that the swarm as a whole interacts inelastically with the boundaries.

- In the channel with periodic boundary conditions along the channel direction, we find the swarming solution previously described for periodic domains by [18] and match their velocity to the von Mises distribution theoretically derived for the macroscopic Vicsek Model by [10]. In addition, we find a new type of solution for the Vicsek Model corresponding to a periodic solution similar to a shear flow where the particles move in opposite directions along the channel wall and the majority of the particles generate a localized high density swarm that moves from one boundary to the other and back, changing their flow direction parallel to the wall.

- A swarm moving in a disk has spatial coherence and moves around the boundary of the disk. The coherence increases as the noise level decreases asymptotically leading to a $\delta$ - density distribution. The most notable feature of this periodic solution is that its time averaged density is numerically identical to the density of a mill solution of the macroscopic Vicsek Model, restricted to the disk. This suggests the open problem to prove under what conditions the time average of a particle model simulation becomes the equilibrium solution of a macroscopic model that is derived for large time and spatial scales.

- Simulations in square domains lead to solutions that are structurally similar to those occurring in the disk: Coherent swarms break the D4 symmetry of the square and start to rotate. The center of mass describes a closed trajectory that is rotationally symmetric but not 
reflection symmetric ( $C^{4}$-symmetry). When the noise is too large, the rotational pattern is lost and particles are seen evenly dispersed in the domain. The time averaged density profile shows the particles more concentrated at the boundary for small noise. When noise is extremely small, swarms are polarized when they move between boundaries but lose polarization when the swarm hits a boundary. As a result the trajectory of the center of mass appears chaotic.

\section{Acknowledgement}

All three authors gratefully acknowledge support through NSF grant DMS-1515592 and travel support through the KI-Net grant, NSF RNMS grant No. 1107291.

[1] M. Aldana, V. Dossetti, C. Huepe, V. M. Kenkre, And H. LARRALDE, Phase transitions in systems of self-propelled agents and related network models, Physical review letters, 98 (2007), p. 095702.

[2] I. Aoki, A simulation study on the schooling mechanism in fish., Bulletin of The Japanese Society of Fisheries Science, 48 (1982), pp. 10811088 .

[3] M. Ballerini, N. Cabibbo, R. Candelier, A. Cavagna, E. Cisbani, I. Giardina, V. Lecomte, A. Orlandi, G. Parisi, A. ProCACCINI, ET AL., Interaction ruling animal collective behavior depends on topological rather than metric distance: Evidence from a field study, Proceedings of the national academy of sciences, 105 (2008), pp. 12321237.

[4] H. Chaté, F. Ginelli, G. Grégoire, F. Peruani, and F. RayNAUD, Modeling collective motion: variations on the vicsek model, The European Physical Journal B, 64 (2008), pp. 451-456.

[5] I. D. Couzin, J. Krause, R. James, G. D. Ruxton, and N. R. FRANKS, Collective memory and spatial sorting in animal groups, Journal of theoretical biology, 218 (2002), pp. 1-11.

[6] F. Cucker and S. Smale, Emergent behavior in flocks, Automatic Control, IEEE Transactions on, 52 (2007), pp. 852-862. 
[7] A. Czirók, A.-L. Barabási, and T. Vicsek, Collective motion of self-propelled particles: Kinetic phase transition in one dimension, Physical Review Letters, 82 (1999), p. 209.

[8] A. Czirók And T. VicseK, Collective behavior of interacting selfpropelled particles, Physica A: Statistical Mechanics and its Applications, 281 (2000), pp. 17-29.

[9] P. Degond, A. Frouvelle, And J.-G. Liu, Macroscopic limits and phase transition in a system of self-propelled particles, Journal of nonlinear science, 23 (2013), pp. 427-456.

[10] P. Degond And S. Motsch, Continuum limit of self-driven particles with orientation interaction, Mathematical Models and Methods in Applied Sciences, 18 (2008), pp. 1193-1215.

[11] M. R. D'Orsogna, Y.-L. Chuang, A. L. Bertozzi, and L. S. CHAYES, Self-propelled particles with soft-core interactions: patterns, stability, and collapse, Physical review letters, 96 (2006), p. 104302.

[12] H. Fehske, R. Schneider, And A. Weisse, Computational ManyParticle Physics, Springer Verlag, 2007.

[13] A. Frouvelle And J.-G. Liu, Dynamics in a kinetic model of oriented particles with phase transition, SIAM Journal on Mathematical Analysis, 44 (2012), pp. 791-826.

[14] J. Gautrais, F. Ginelli, R. Fournier, S. Blanco, M. Soria, H. Chaté, and G. Theraulaz, Deciphering interactions in moving animal groups, PLoS Comput Biol, 8 (2012), p. e1002678.

[15] G. GréGoire And H. Chaté, Onset of collective and cohesive motion, Physical review letters, 92 (2004), p. 025702.

[16] G. Grgoire and H. Chat, Onset of Collective and Cohesive Motion, Physical Review Letters, 92 (2004), p. 25702.

[17] P. W. Miller and N. T. Ouellette, Impact fragmentation of model flocks, Physical Review E, 89 (2014), p. 042806. 
[18] S. Motsch And L. NAVOret, Numerical simulations of a nonconservative hyperbolic system with geometric constraints describing swarming behavior, Multiscale Modeling \& Simulation, 9 (2011), pp. 1253-1275.

[19] C. W. Reynolds, Flocks, herds and schools: A distributed behavioral model, ACM Siggraph Computer Graphics, 21 (1987), pp. 25-34.

[20] D. Strömbom, Collective motion from local attraction, Journal of theoretical biology, 283 (2011), pp. 145-151.

[21] T. Vicsek, A. Czirók, E. Ben-Jacob, I. Cohen, And O. SHOCHet, Novel type of phase transition in a system of self-driven particles, Physical review letters, 75 (1995), p. 1226.

[22] T. Vicsek and A. Zafeiris, Collective motion, Physics Reports, 517 (2012), pp. $71-140$. 